\title{
Contributing Reviewers in 2017
}

J Neuroanaesthesiol Crit Care 2018;5:i.

The Journal of Neuroanaesthesiology and Critical Care wishes to recognize all those who contributed as an expert peer reviewer of scientific articles submitted in 2017.

Thank you for your contributions to the Journal of Neuroanaesthesiology and Critical Care in 2017.

Arvind Arya

Ashish Bindra

Ashok Badhe

Barkha Bindu

Bhadri Narayan

Bhagya Ranjan Jena

Charu Mahajan

Dhritiman Chakrabarti

Dilip Kulkarni

G. Parameswara

Gaurav Tomar

Girija Rath

Gyaninder Singh

Hari Dash

Hemangi Karnik

Hemant Bhagat

Indu Kapoor

Joseph Monteiro

Kiran Jangra
Lakshmi Venkatraghvan

Mani Kalaivani

Mary Abraham

Monica Tandon

Radhakrishnan M.

Navdeep Sokhal

Nidi Gupta

Nidhi B. Panda

Parmod Bithal

Pragati Ganjoo

Prasanna Bidkar

Rajeeb Kumar Mishra

Rajiv Chawla

Rakesh Garg

Ramamani Mariappan

Ramesh V. J.

Ravindra Mohan Pandey

Ritesh Lamsal

S. Manikandan
Satyen Parida

Shashi Srivastava

Shobha Purohit

Siddharth Chavali

Somnath Bose

Sonia Bansal

Sriganesh Kamath

Srilata Moningi

Surya Dube

Swagata Tripathy

Tumul Chowdhury

Umamaheswara Rao

Varun Jain

Vasudha Singhal

Venkatesh Kesavan

Vikas Chauhan

Zulfiqar Ali
DOI https://doi.org/ 10.1055/s-0038-1624064. ISSN 2348-0548.
Copyright $\odot 2018$ Indian Society of Neuroanaesthesiology and Critical Care
License terms

(우 (1) $\Theta \circledast$ 
